\title{
Selecting the Appropriate Decision Alternatives using SM and AHP
}

\author{
Ahmad A. Maidamisa ${ }^{1}$ Rohanin Ahmad ${ }^{2} \quad$ Abdul. A. M. Ismail ${ }^{3}$ \\ ${ }_{123}$ Department of Mathematics \\ Universiti Teknologi Malaysia \\ 81310 UTM Johor Bahru
}

\begin{abstract}
The paper employed two decision analysis methods to solve a farmer's choice of the crop's problem, for planting purposes. The first method is scoring model, while the second method is the analytical hierarchy process. A result yields two different ranking of crops. It was shown that the ranking obtained from the first method has no association with the ranking produced by the second method. This paper is the first research work to utilize scoring method with the analytical hierarchy process in Agricultural decision making. It compares two rankings results and infers on their performance.
\end{abstract}

General Terms: Multi criteria decision analysis techniques.

Keywords: Analytic Hierarchy Process, Scoring Model, Ranking.

\section{INTRODUCTION}

The use of qualitative judgment in multi criterion decisionmaking is receiving increasing attention, and a variety of methods have been developed that cover a wide range of techniques. One method that has received increasing attention and used in papers is the relatively recent developed Scoring Model (SM) [1] and Analytic Hierarchy Process (AHP) [2]. These two methods have been widely documented in a variety of problem areas. Except for few cases, these qualitative decision-making techniques have not been used extensively in agricultural decision-making in developing countries. The paper briefly applied SM and AHP in crop selection problem.

Gurusami [3] employed SM to select a supplier among three suppliers, whom will set a performance targets for future and current model of automobiles for Ford Motor Company? AHP has been widely applied in diverse areas of agriculture. Visage [4] used linear programming and SM in agriculture decisionmaking in South Africa. AHP is a decision aiding method developed by Saaty [2]. It aims at qualifying relative's priorities for a given set of alternatives on a ratio scale, based on the judgment of the decision maker, and stresses the importance of the intuitive judgments of a decision maker as well as the consistency of the comparison of alternatives in the decision-making process. Since a decision maker bases judgment on knowledge and experience and then makes a decision accordingly, the AHP approach agrees well with the behavior of a decision maker.

Kamal et al. [5] used AHP to select the most to qualify a contractor at the pre-qualification stage. A hierarchical structure of the contractor is constructed for the pre qualification criteria. The pre qualification criterion is prioritized. A descending order list of the contractor is created in order to select the best. Finding has shown that among the six attributes considered experience is the most suitable follow by financial stability for the selection criteria process $(37.2 \%$ and $29.3 \%$ respectively). AHP has been widely applied in diverse areas of agriculture. Alphonse [6] employed AHP to review some potential area of application in agricultural practice in developing countries. By selecting the best crop which is to be planted in a given piece of land. Results have shown that $17.7 \%$ of the land are to be allocated to Maize $45.8 \%$ is to be allocated to Millet and $45.6 \%$ of the land is to be allocated to Cassava.

Bhatta and Doppler [7] applied AHP to look into the prevailing factor of farming differentiation in the rural-urban interface of the densely populated Kathmandu valley in Nepal. There are certain factors that cause farming variation in the interfaces. These factors were incorporated into the AHP model and then subjected to farmers' judgment in distinctly delineated three farming zones. Findings reveal that rural inaccessibility is the most influential factor of subsistence farming. Harvesting higher produce is the primary booster of inorganic farming. Agro ecological consideration is the principal motive towards the small holder organic production.

Moghaddam and Karami [8] utilize AHP to select an appropriate agricultural development program in Iran. They applied AHP to determine the priority of two theories based on sustainable agricultural development's models. Results have shown that wise use of resources and environmental protection and product quality are the most important criteria for sustainable agriculture of Iran. Furthermore, a check is conducted to determine the critical factors that affect the priority of decision alternatives.It was revealed that ecological modernization based sustainable agricultural development model has a higher priority as the theoretical base of agricultural development of Iran.

Montazar and Zadbagher [9] used AHP in measuring the global water production status in an irrigation network. There are 14 major irrigation networks with 14 attributes to be examined using AHP. The networks serve as the decision alternative to be rank cordially. An irrigation network is ranked efficient, if its score is at least $60 \%$, otherwise it is ranked semi efficient. Results have shown that Dez irrigation network is the highest, while Saveh is the lowest in terms of global water production. The AHP result is judged using fiveyear average field investigation. This Model result has agreed with the results determined from the field survey.

Nandi et al. [10] has shown that AHP can be applied during project selection phase. It can be used to examine and evaluate the project viability during bidding stage in order to 
overcome the limitations of the traditional approach currently used by contractors. In this study, AHP is used to assist a contracting firm in measuring the advantage and disadvantages of a project and help it decide whether to bid for a project or not to bid. The results have shown that a contractor can bid for the project that has the highest priority if he wants to bid for only one project.

The analysis presented in this paper consists of a three-stage approach. The first stage is solving a crop selection problem by SM. The second stage is solving the crop selection problem by AHP. The last stage is comparing the first-stage result with the second-stage results to deduce a conclusive statistical inference. The paper is organized as follows. In the next section, the problem statement is highlighted. In the third section multi criteria decision is provided. The fourth section provides the simulations and results. In the fifth section, the paper is summarized and a final conclusion is drawn.

\section{PROBLEM STATEMENT}

A farmer likes to determine on the portions of his farm land that should be allocated for agricultural purposes, to the following crops are Palm Oil (PO), Rubber (RB), Timber (TB), Cocoa (CO), Cocoa Nut (CN), Rice (RC), and Banana (BA). The following criteria are considered for this decision problem. The farmer sources of livelihood (FLH), a limited cash expenditure on labour (LEL), gross farm income (GFI), and sources of raw material (RMS). What portion of farm land is to be set aside for each of these crops? Which crop should be given priority? The paper considers two MCDM approaches to solve the farmer decision problem. The first is Scoring Model(SM). The second is Analytical Hierarchy Process (AHP). Detail elaboration on these models is given below.

\section{MULTI CRITERIA DECISION MAKING}

Multiple-criteria decision-making (MCDM) or multiplecriteria decision analysis (MCDA) is a sub-discipline of operation's research that explicitly considers multiple criteria in decision-making environments. There are generally multiple conflicting criteria that need to be evaluated in making decisions in our daily lives or professional backgrounds. Cost or price is commonly one of the principal criteria. Some measure of quality is mostly another criterion that conflicts with the cost. In purchasing a car, cost, comfort, safety, and fuel economy may be some of the primary criteria we consider. It is unusual to have the cheapest car to be the most comfortable and the safest. In portfolio management, we are interested in getting high returns but at the same time reduce our risks. Again, the stocks that have the potential of bringing high returns to typically also carry high risks of losing money. In the service industry, customer satisfaction and the cost of providing service are two conflicting criteria that would be useful to consider.

Experiences have shown that we normally consider multiple criteria implicitly, and we may be comfortable with the effects of such decisions that are made based on only intuition. On the other hand, when interests are high, it is important to properly structure the problem and explicitly evaluate multiple criteria In making the decision of whether to build a nuclear power plant or not, and where to build it, there are not only very complex issues involving multiple criteria, but there are also multiple parties that are deeply involved in the effects. A structuring complex problem well and considering multiple criteria explicitly, leads to more informed and better decisions. There have been important advances in this area since the start of the modern multiple criteria decision making discipline in the early 1960s.

\subsection{Scoring Model}

Scoring Model (SM) is a relatively quick and easy way to identify the best decision alternative for a multi criteria decision problem. Equation (1) is used to compute scores denoted as.

$$
S_{j}=\sum w_{i} r_{i j}
$$

where

$$
\text { alternative } j
$$

$$
\begin{aligned}
& S_{j}=\text { score for decision alternatives } j . \\
& w_{i}=\text { weight for criterion } i \\
& r_{i j}=\text { rating for criterion } i \text { and for decision }
\end{aligned}
$$

Weights are subjectively assigned to each criterion to indicate the criterion relative importance in the decision-making process. For example, using a five-point scale, the question used to assign a weight to the farmer sources of livelihood criteria would be as in Table 1. Relative to the other criteria we are considering, how important is livelihood? By repeating this question for each of the other four criteria, the farmer is provided with the criterion weights as shown in Table 1 .

Table 1 Weights for the four decisions criteria

\begin{tabular}{llc}
\hline Criteria & Importance & Weight $\left(w_{i}\right)$ \\
\hline FLH & Very important & 5 \\
LEL & Average important & 3 \\
GFI & Somewhat important & 4 \\
RMS & Average important & 3 \\
\hline
\end{tabular}

\subsection{Analytical Hierarchy Process}

Analytical Hierarchy Process (AHP) is a multi criteria decision sustains tool that aims at solving complex decision problems. It was originally developed by Saaty [2]. The important characteristic of AHP is that it converts individual preferences into ratio scale weights thereby providing the avenue for effective comparison and ranking of the decision factors. The fundamental ascriptions of AHP are the hierarchical structure of complexity,pair-wise comparisons, redundant judgments, an eigenvector method for deriving weights and consistency considerations. Like many methods, it allows decision-makers to create a model of a complex problem with the goal at the top and criteria, sub-criteria and decision alternatives at levels in the drop-down manner [11]. 
As four criteria were selected for finding their influence on the predominance of a particular farmer's choice, the dimension of the matrix, therefore, is $4 \times 4$. It is because one column and one row correspond to each factor and there were 6 pair-wise comparisons using the formula. $n(n-1) / 2$. If this matrix is denoted as $\Omega=\left(\eta_{i j}\right)$ where $\eta_{i j}$ is the element of $i^{\text {th }}$ row and $j^{\text {th }}$ column of the matrix, all its entries are obtained by inscribing the relative importance of each criterion over another with respect to the goal. Pair-wise comparison can be performed by assigning an integer ranging from 1 to 9 or the reciprocal of such an integer to each cell of the matrix to measure the relative importance of the factors that characterize the cell. To fill the lower triangular matrix, the reciprocal values of the upper diagonal are used as. $\eta_{i j}=1 / \eta_{i j}$, where $\eta_{i j}>0$.

In the AHP matrix $\Omega$, the cells along the diagonal are given the value of 1 , so that the row factor compared to the same column factor receives a unit value. The cell representing two different factors in row and column with equal contribution or influence also gets a unit value. The user has to realize that this choice is itself a statement of relative value. The AHP approach assumes that each of the factors under assessment is independent. It allows some small inconsistency in the judgment because human responses are not always consistent. In practice $100 \%$ consistency is difficult to achieve, but the method can still be applied when there is some degree of interdependence. For calculating the index of inconsistency, AHP uses the consistency ratio (CR). A value of inconsistency index is lower than $10 \%$ is acceptable, particularly if matrix is $4 \times 4$ above. In certain cases, although higher value of inconsistency index requires re-evaluation of the pair wise comparisons, decisions obtained in that kind of situation could also be taken as the best alternative. Consistency index is calculated as $\mathrm{CI}=\left(\lambda_{\max }-n\right) /(n-1)$.

The CI of a randomly generated reciprocal matrix is called random index (RI). An average RI for the matrices of order 115 can be found in [11]. The CR can hence be calculated as the ratio of $\mathrm{CI}$ to $\mathrm{RI}$ i.e. $(\mathrm{CR}=\mathrm{CI} / \mathrm{RI})$. The first step in AHP is to develop a graphical representation of the problem in terms of the overall goal, criteria and decision alternatives.

Figure 1 shows the hierarchy for the farmer's decision problem. The top level of the hierarchy gives the overall goal i.e. to determine the portion of the farm land to allocate to a particular crop. The second level shows the four criteria that contribute to the achievement of the overall goal. The seven decision alternatives Palm Oil; Rubber, Timber, Cocoa, Cocoa nut, Rice and Banana are shown at the third level.

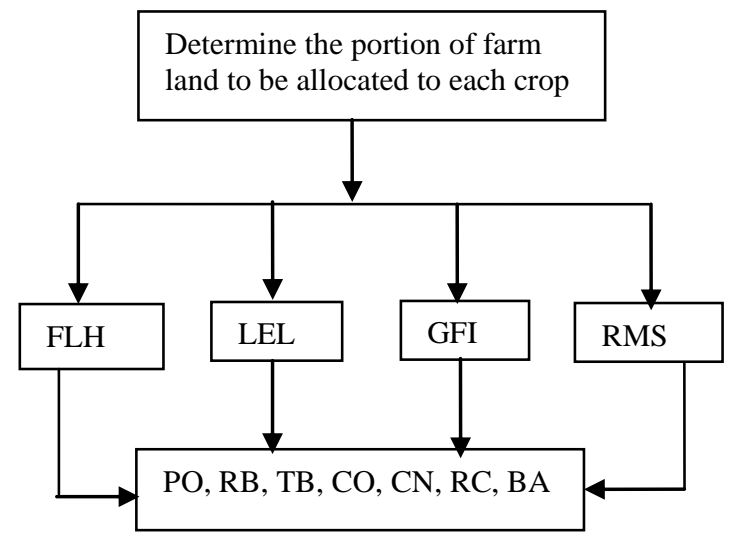

Fig: 1 Hierarchy for the portion determination problem

In order to apply AHP, the decision maker must specify his judgments of the relative importance of each criterion's contribution towards achieving the overall goal. The evaluation will be elicited using questions such as "given the two criteria farmers' livelihood and gross farm income, which one is more important in determining the portion of the farm land to be allocated to each crop? How important? ". Similar pair wise comparisons for other criteria can be done to generate the pair wise comparison matrix.

The decision maker (farmer) believes, for example, that farmer's livelihood is very strongly to extremely more prefer than the gross farm income. As a result gross farm income is rated 8 as preferred to farmer's livelihood, as indicated above the rest of the matrix is filled in a similar manner.

$\Omega=\left[\begin{array}{cccc}1 & 4 & 8 & 9 \\ 1 / 4 & 1 & 2 & 3 \\ 1 / 8 & 1 / 2 & 1 & 2 \\ 1 / 9 & 1 / 3 & 1 / 2 & 1\end{array}\right]$

\section{SIMULATIONS AND RESULTS}

In this section simulation experimentation is conducted using SM, AHP and statistical analysis. The result is presented below.

\subsection{SM}

Table 2 shows the computation using SM. The highest score is 97, which corresponds to palm oil. Next to it is 95, which corresponds to cocoa. Timber is ranked third with a score of 94. Rice is ranked fourth with score of 82 . The fifth is rubber with a score of 78. Banana score is 76 and lastly cocoa nut is ranked 71 . Thus palm oil is the recommended decision.

Table 2 Computation of scores for the seven decision alternatives

\begin{tabular}{|c|c|c|c|c|c|c|c|c|}
\hline \multicolumn{3}{|c|}{ Weight PO } & $\overline{R B}$ & $\mathrm{CO}$ & \multicolumn{2}{|c|}{$\mathrm{CN}$} & $\bar{C}$ & $\overline{\mathrm{BA}}$ \\
\hline Criteria & $\left(w_{i}\right)$ & $w_{i} r_{i 1}$ & $w_{i} r_{i 2}$ & $w_{i} r_{i 3}$ & $w_{i} r_{i 4}$ & $w_{i} r_{i 5}$ & $w_{i} r_{i 6}$ & $w_{i} r_{i 7}$ \\
\hline FLH & 5 & 45 & 30 & 40 & 45 & 30 & 25 & \\
\hline LEL & 3 & 9 & 15 & 15 & 12 & 9 & 12 & \\
\hline GFI & 4 & 28 & 12 & 24 & 20 & 20 & 24 & \\
\hline RMS & 3 & 15 & 21 & 15 & 18 & 12 & 21 & \\
\hline
\end{tabular}




\subsection{AHP}

Using Micro-Soft Excel for computation, the preferences matrix $\Omega$, serves as inputs and the eigenvector $w_{i}$ as output. Hence, the consistency ratio is calculated as follows.

$\bar{w}=\left[\begin{array}{c}- \\ w_{F L H} \\ - \\ w_{L E L} \\ - \\ w_{G F I} \\ - \\ w_{R M S}\end{array}\right]=\left[\begin{array}{l}0.664 \\ 0.178 \\ 0.098 \\ 0.061\end{array}\right], \lambda_{\max }=4.056$

$\mathrm{C} \mathrm{I}=0.019, \mathrm{C} \mathrm{R}=0.021$.

The consistency ratio is the ratio of the decision makers' inconsistencies obtained from randomly generated preferences. The next step is to make pair-wise comparisons for the seven crops alternatives with respect to each of the criteria. We illustrate this with respect to limited cash expenditure on labors the second attribute. $\Omega=\left[\begin{array}{ccccccc}1 & 2 & 3 & 3 & 4 & 3 & 8 \\ 1 / 2 & 1 & 3 & 3 & 3 & 3 & 7 \\ 1 / 3 & 1 / 3 & 1 & 2 & 2 & 1 / 3 & 2 \\ 1 / 3 & 1 / 3 & 1 / 2 & 1 & 2 & 2 & 2 \\ 1 / 4 & 1 / 3 & 1 / 2 & 1 / 2 & 1 & 3 & 2 \\ 1 / 3 & 1 / 3 & 3 & 1 / 2 & 1 / 3 & 1 & 2 \\ 1 / 8 & 1 / 7 & 1 / 2 & 1 / 2 & 1 / 2 & 1 / 2 & 1\end{array}\right]$ $-\bar{w}=\left[\begin{array}{l}0.317 \\ 0.243 \\ 0.105 \\ 0.103 \\ 0.093 \\ 0.098 \\ 0.041\end{array}\right], \lambda_{\max }=7.733, C I=0.122$,

$\mathrm{CR}=0.093$.

Here, the farmer he believes that palm oil is strongly more preferred than cocoa. Similar pair wise comparison must be made with respect to each of the four attributes. The resulting set of weights for each of the alternatives with respect to each criterion is presented in the following matrix below.

$\left[\begin{array}{lllllll}0.317 & 0.243 & 0.105 & 0.103 & 0.093 & 0.098 & 0.041 \\ 0.038 & 0.050 & 0.154 & 0.133 & 0.162 & 0.177 & 0.287 \\ 0.357 & 0.233 & 0.102 & 0.095 & 0.088 & 0.087 & 0.039 \\ 0.036 & 0.060 & 0.135 & 0.128 & 0.170 & 0.182 & 0.290\end{array}\right]$

Finally, the portions of farm land to be allocated to each crop is found by determining the product of each criteria priorities with respect to the weights as given below.

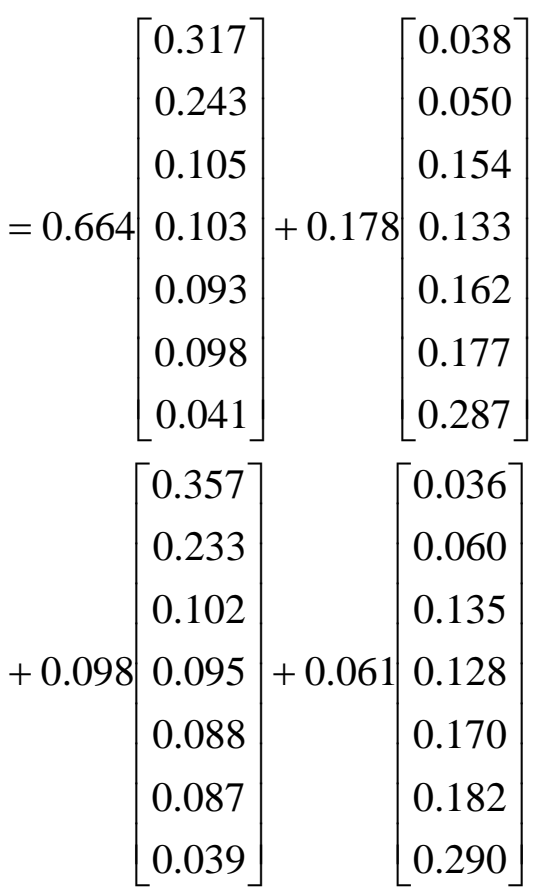

\begin{tabular}{|c|c|}
\hline$P O$ & $25.44 \%$ \\
\hline$R B$ & $19.67 \%$ \\
\hline$T P$ & $11.54 \%$ \\
\hline$C O=$ & $10.92 \%$ \\
\hline & $10.96 \%$ \\
\hline & $11.62 \%$ \\
\hline & 9.989 \\
\hline
\end{tabular}

The composite AHP score indicates that according to the realities facing the farmer is to allocate about $25.44 \%$ of the farm land to Palm oil, $19.67 \%$ to rubber, $11.54 \%$ to Timber, $10.92 \%$ to Cocoa, $10.96 \%$ to Cocoa nut, $11.62 \%$ to rice and $9.98 \%$ to Banana cultivations.

\subsection{Comparison between SM and AHP}

Table 3 shows the portion of land in numerical terms which is set aside for specific type of crop. For instance, $25.44 \%$ of farm lands are a reserve for palm oil cultivation. $10.92 \%$ of farm land is a reserve for cocoa farming and so on.

Table 3 Land appropriation

\begin{tabular}{lc}
\hline SM & AHP \\
\hline PO & $25.44 \%$ \\
CO & $10.92 \%$ \\
TB & $11.54 \%$ \\
RC & $11.62 \%$ \\
RB & $19.67 \%$ \\
BA & $9.98 \%$ \\
CN & $10.96 \%$ \\
\hline
\end{tabular}


The efficacy of the two methods is determined by testing the following hypothesis below. Spearman's rank correlation coefficient is used to test the following alternatives.

$H_{0}$ : There is no association between SM and AHP

$H_{1}$ : There is an association between SM and AHP

The spearman Rho Equation is given as.

$r_{s}=1-\frac{6 \sum d^{2}}{n\left(n^{2}-1\right)}$

(2)

where

$r_{s}=$ Spearman rank order correlation coefficient

rho

$$
\begin{aligned}
& d=\text { Difference between ranks } \\
& n=\text { Number of observations }
\end{aligned}
$$

Using Equation (2) the correlation coefficient is computed as $r_{s}=0.4286$. from the critical Spear man Rho Table [12] at $5 \%$ level of significance the value is $r_{s}=0.7860$. since the tabulated value is greater than the computed value i.e. $r_{s}=0.4286 \leq r_{0.05}=0.7860$.

Therefore we accept $H_{0}$ and conclude that there is no association between SM and AHP.

\section{SUMMARY AND CONCLUSION}

There are many operation's research methods to aid a farmer in allocating portions of farm land to a number of agricultural crops. These approaches utilize mathematical programming techniques. However, their applicability is limited due to lack of data and their inability to handle qualitative issues. SM and AHP have to avoid these limitations. The computation presented here are not intended to be an "answer" to the farmers' decision problem but just to illustrate the steps involved when using the SM and AHP. In this conclusion, AHP has set aside the portion of farm land, whereas SM has selected the type of crops for cultivation purposes.

\section{REFERENCES}

[1] Anderson, D., Sweeney, D. and Williams, T. 2008. An introduction to management science: Quantitative approaches to decision making. Australia. Cengage learning.

[2] Saaty, T. 2007. The analytic hierarchy process. New York, McGraw-Hill.

[3] Gurusami, A.S. 2009. Fords wrenching decision. OR/MS Today, 5, 36-39.

[4] Visagie, S. 2004. Operations research in agriculture. ORSSA/ONSA Newsletter. 4(2), 5-7.

[5] Kamal, M. 2001. Application of the AHP in project management. Int. Journal of project management. 19(1), 19-27.

[6] Alphonce, C. 1997. Application of analytical hierarchy process in agriculture in developing countries. Agricultural systems, 53, 97-112.

[7] Bhatta, G. and Doppler, W. 2010. Farming differentiation in the rural urban interfaces of the middle mountains Nepal: Application of analytical hierarchy process modelling. Journal of agricultural sciences. 2(4), 37-51.

[8] Moghaddam, K. and Karami, E. 2008. A multi criteria evaluation of sustainable agricultural development models using AHP. Environmental development sustainability. 10(1), 407-426.

[9] Montazar, A. and Zadbagher, E. 2010. An analytical hierarchy model for assessing global water productivity of irrigation networks in Iran. Water resources manage, 24(1), 2817-2832.

[10] Nandi, S., Paul, S. and Phadtare, M. 2011. An AHP based construction project selection methods. Decision. 38(1), 91-118.

[11] Saaty, T. 2002. Decision making for leaders: The analytical hierarchy decision in a complex world. USA. RWS Publishers.

[12] Sheskin, D. 2007. Handbook of parametric and non parametric statistical procedures. Florida, Champman Hall/CRC. 